\title{
Application and Research on BIM Technology in the Reconnaissance and Design Stage of Engineering
}

\author{
Zhou Ling \\ Xi’An Siyuan University, Shaanxi, China, 710038
}

Keywords: BIM, Information, Reconnaissance and Design, Application and Research

\begin{abstract}
The emergence of BIM technology is a new stage in the information of the construction industry. It provides a platform for information integration and sharing at all stages of the project to better help the participants to work together. This paper describes the concept and feature of BIM technology and then it expounds the application and technical advantages of BIM in engineering reconnaissance and design stage.
\end{abstract}

\section{Introduction}

Information is an important foundation for the development of the construction industry. With the development of engineering technology, the design is more and more complex. The data between different professions is not on the same platform, and collaboration has also become more difficult. BIM technology not only brings advanced technical tools to the construction industry, but also brings advanced cooperation concepts to all participants in the project life cycle.

BIM (Building Information Modeling) is a unified data platform, in the whole life cycle of building engineering. Create a virtual building model containing all building information, with multi-view, parameterized and intelligent buildings. A complete visual 3D environment model is established based on the component. The owners, designers, construction parties and other parties can work together on the BIM platform, such as data lossless transmission, information sharing. And the BIM platform can promote the management of design, construction, operation and other stages. We can improve the quality, reduce costs and increase revenue in the reconnaissance and design stage of engineering.

\section{The feature of BIM technology}

Based on the centralized management of the project entity object process, BIM technology realize the information transmission channel and change the way of information accumulation, and the mode transformation. BIM technology has the characteristics of digital expression function and implementation effect. On the basis of 3d digital technology, people can more intuitively see the architectural design effect. Which is of great help to improve the quality and efficiency of construction projects. BIM platform realizes data information resource sharing and collaboration in the whole construction project. This paper summarizes the technical characteristics of BIM technology as follows.

\subsection{Visualization}

At present, the 3D design discussion and the phased renderings mainly use 3Dmax. There is a big gap with the traditional design. A lot of design work is still using flat, elevation, and profile to showcase the design results based on the CAD. However, more and more large-scale projects and heterosexual buildings designed by 2D are very difficult. Visualization technology enables people to see the design effect more vividly.

\subsection{Collaboration}

The lack of a unified information standard is very painful for many designers. BIM technology transforms the previous drawing mode into a form of building a model, allowing different 
professions and designers to add, modify, and store different building information. And keep the model updated and unified in real time. The current BIM data standard commonly used and applied in the field of construction engineering is the IFC standard developed by the International Collaborative Working Union.

\subsection{Simulative}

BIM technology can be used to simulate energy conservation assessment, property management and other follow-up sustainable design, and can also be used in the construction stage. Project managers can plan and control the construction management process from a global perspective through simulation of BIM technology. By coordinating the interaction of various activities in the construction process, the project manager can achieve comprehensive management, such as ensuring the construction period, quality, cost control, communication and coordination.

\subsection{Optimization}

BIM technology can optimize project plan, combine project design and cost management, track the impact of design on investment value-added in real time, which is helpful for improving the efficiency and level of cost management. By comparing different schemes, decision-makers can choose the optimal scheme by comparison. The plan can also optimize the design and construction of mega projects, which can bring significant time and cost improvements.

\subsection{Convenience}

BIM technology is a database that storing all the information, extracting the required data at any time, and forming a virtual 3D building model, which can help the designer and the owner to generate the required drawings, inspection reports and improvement plans. The design efficiency has been greatly improved.

\section{The development status of BIM technology in the reconnaissance and design stage}

BIM Technology originated in the United States in the 1970s. The concept was proposed by Dr. Chuck Eastman of Georgia tech. In view of the changes brought by BIM technology to the development of the construction industry and its technology, BIM technology has been widely promoted and has been widely used internationally.

In 2002, the country began to introduce BIM technology and management methods, and the government and industry associations also issued relevant policies to promote the popularization of BIM technology. Due to the late start, it is still in the research and promotion stage, and is mainly used in the reconnaissance design and construction stage. With the continuous exploration and development of the domestic architectural field, the traditional reconnaissance and design uses the 2D model construction in CAD. However, there are often errors, omissions, collisions, and missing phenomena.

BIM technology can transform the traditional 2D model into 3D. The model optimizes the design scheme through data analysis. At present, it is widely used in various aspects of geological exploration, pipeline synthesis, performance analysis, coordinated design, engineering cost and other reconnaissance and design, which improves the efficiency of reconnaissance and design. However, the current projects that use BIM technology in China are mainly landmarks, demonstrations, and large-scale buildings. The popularity is low, and they are mainly concentrated in the reconnaissance and design stage. The scope involved is small, and there is a big gap with the international.

\section{Application of BIM technology in reconnaissance and design}

\subsection{Geological Prospecting}

Geological exploration is the primary link of architectural design. The application of BIM technology can make geotechnical engineering survey data and information have enough accuracy. 
We can make survey data visualization through BIM technology, and then provide reasonable basic design scheme and geotechnical design parameters. At the same time, we can simulate and optimize the underground project construction scheme. So that we can more easily realize the 3D integration of the above ground-building and underground-space geological information, which will help effectively avoid engineering risks and create the best economic and social benefits.

\subsection{Pipeline synthesis}

BIM synthesis technology can gather professional information of architecture, structure and electromechanical engineering. We can intuitively find the collision problem of design through 3D model, which greatly helps to improve overall efficiency and pipeline design capabilities. At the same time, it reduces the complexity of construction, cost and time waste.

\subsection{Performance analysis}

The performance analysis of BIM technology can be used for energy consumption assessment, lighting analysis and HVAC design, which is helpful for performance analysis, such as, ensuring the consistency of building performance data, compressing the design cycle, improving the design quality, and so on. At the same time, Reconnaissance and design enterprises can provide owners with more professional and thoughtful cooperation and services.

\subsection{Collaborative Design}

Collaborative design is a new form of architectural design, which can realize the sharing of architectural engineering information on the same professional platform through the core model of BIM. With the gradual accumulation and improvement of data information in each stage, the technical content of collaborative design will be greatly improved.

\subsection{Project cost}

Using BIM to calculate the project quantity, it can provide the required engineering quantity information for the engineering cost profession. Through quick summary and statistics, the project manager can ensure that the project summary information is completely consistent with the original design results. BIM technology can reduce the intensity of foundation work by improving calculation efficiency and ensuring calculation accuracy. The application of BIM in engineering cost can better face design changes and facilitate the control of project cost.

\section{Conclusions}

The advantages of BIM technology have shown its suitability and necessity in the field of architectural design. The reconnaissance and design stage is the most important stages of construction engineering, which determines the future architecture. BIM technology can better improve the knowledge management and project management capabilities, which can help reconnaissance and design units to provide consulting or participation in the construction phase, expand service areas and boundaries, enable reconnaissance and design information throughout the project lifecycle. BIM technology will become a necessity for the future development of modernization, promote the rapid upgrading, and create more technical and economic value.

\section{Acknowledgements}

《Practice and Research on the Individual Test Method of Experimental Courses in Applied Private Colleges and Universities》(Project Number: SGH18H532)

\section{References}

[1] Guo Junli, Teng Jiaying, Wu Xianguo. Research on collaborative management method of IPD construction project based on BIM [J]. Construction Technology, 2012 (22): 81-85. 
[2] Wang Tingkui, Deng Wei, Li Wei. A review of the application research of BIM design stage based on content analysis. [J] Construction Economy, 2016 (09): 100 105.

[3] Zhang Qin. BIM technology promotes the transformation of the construction industry [J]. Fujian architecture, 2011, (6) : 119-120.

[4] China survey and design association .practical BIM implementation framework [M]. Beijing: China building industry press, 2010. 